\title{
Prediction of Type 1 Diabetes
}

\author{
Henry A. Erlich, ${ }^{1}$ Ana M. Valdes, ${ }^{2}$ and Janelle A. Noble ${ }^{3}$
}

T ype 1 diabetes (T1D) is a chronic autoimmune disorder in which the destruction of the insulinproducing cells and resulting clinical symptoms are preceded by the appearance of a number of islet-cell specific autoantibodies. Linkage (1) and association analyses have demonstrated that type 1 diabetes has a very strong genetic component, with specific alleles and haplotypes at the HLA class II genes, as well as HLA-A and $-B$ alleles, conferring either susceptibility to or protection from T1D (2-6). The ability to identify individuals at high risk for type 1 diabetes using genetic and/or autoantibody markers (7-10) has been a long-standing goal of the diabetes research and clinical community and a critical element in T1D prevention strategies. The role of prediction in prevention is twofold: 1) Clinical trials to evaluate potential preventative interventions are more efficient if the recruited subjects are at high T1D risk, and 2) interventions are likely to be more effective if administered early in disease progression or during the prediabetic phase, a stage identified by autoantibody markers in individuals who carry genetic risk alleles.

Although a large number of genetic variants associated with T1D have been identified by genome-wide association study analyses (11), the major genetic determinants remain specific alleles at the HLA class II and, to a lesser extent, class I loci. Because specific combinations of alleles at the HLA loci determine the genetic susceptibility, the risk for T1D is best captured by considering DR-DQ haplotypes and genotypes rather than alleles at individual loci. The highest-risk T1D genotype is the DRB1*03:01DQA1*05:01-DQB1*02:01/DRB1*04-DQA1*03:01-DQB1*03:02 heterozygote (often expressed using the old serological designation as DR3/DR4 or DQ2/DQ8), with an odds ratio of 30 (2). The increase in risk of this heterozygote compared with the two (DR3/DR3 and DR4/DR4) homozygotes has been attributed to the two transcomplementing DQ heterodimers, including the $\alpha$ (DQA $1 * 05: 01)$ and $\beta$ (DQB1*03:02) heterodimer, present only in this heterozygote (2). Prospective studies of HLA-typed general population samples and first-degree relatives (FDRs; siblings and offspring) have shown that the risk for DR3/DR4 (or DQ2/DQ8) in an FDR is greater than the risk for the same genotype in the general population (12), suggesting that additional loci either within or outside the HLA region also contribute to T1D risk. Among the FDRs, DR3/DR4 siblings

From ${ }^{1}$ Roche Molecular Systems, Pleasanton, California; the ${ }^{2}$ Department of Genetics and Molecular Medicine, School of Medicine, King's College London, London, U.K.; and the ${ }^{3}$ Children's Hospital Oakland Research Institute, Oakland, California.

Corresponding author: Henry A. Erlich, henry.erlich@roche.com.

DOI: $10.2337 / \mathrm{db} 12-1593$

(C) 2013 by the American Diabetes Association. Readers may use this article as long as the work is properly cited, the use is educational and not for profit, and the work is not altered. See http://creativecommons.org/licenses/by -nc-nd/3.0/ for details.

See accompanying brief report, p. 1345. have a greater risk than offspring, and DR3/DR4 siblings who share two HLA haplotypes with the proband have an extremely high risk (12). The incorporation of additional non-HLA genetic markers, such as PTPN22 or INS, into the predictive algorithm can help refine risk estimates, particularly for the DR3/DR4 individuals in the general population (13).

Using the DR3/4 genotype (rather than DR3 or DR4) as a predictive marker will identify individuals at high genetic risk; however, DR3/DR4 individuals represent only around $20-40 \%$ of future T1D cases. In general, a "trade-off" exists between the proportion of future cases identified by the markers (sensitivity) and the positive predictive value for individuals achieved with a broad (genetic, immunological, metabolic) marker panel (specificity) (14,15) (Fig. 1).

Many prospective studies, as well as preventative intervention trials, have focused on FDRs and have also used specific autoantibodies, in addition to genetic markers, for prediction of progression to T1D, for entry into clinical trials, or for studies of environmental triggers (16). Of course, these biomarkers are fundamentally different in that genetic variants identify risk whereas autoantibodies against specific islet-cell antigen markers reflect active and targeted autoimmunity. Of the four commonly used autoantibody assays, antibodies against insulin and glutamic acid decarboxylase appear early during prediabetes, and antibodies to the antigens IA- 2 and ZnT8 appear later; all of these antibodies can be detected well before the onset of clinical diabetes. Typically, autoimmunity in these prospective studies has been defined as a subject testing positive for one or more antibodies on at least two successive visits. Various prediction strategies have combined genetic and autoantibody markers, as well as metabolic markers, in different ways.

In the article by Mbunwe et al. (17), the authors consider various combinations of the IA-2 and ZnT8 autoantibody markers, DR3/DR4 (written in this article as DQ2/DQ8), and HLA-A*24, a class I variant that has been associated with T1D in a variety of studies $(3,6,18)$. Other class I alleles, notably HLA-B*39:06 (susceptible) and B*57:01 (protective) are associated with T1D, but only $\mathrm{A}^{*} 24$ was used in this model. This study selected FDRs who were positive for one or more of the four autoantibodies tested; 288 of these subjects were tested for HLA-A*24 and genotyped for HLA-DQ. The HLA-A*24 typing was performed with a panel of three oligonucleotide probes that could identify but not distinguish all $255 \mathrm{~A}^{*} 24$ alleles, with the exception of a few extremely rare variants. A study of T1D in Filipinos (19) showed that although some A*24 alleles, such as the common HLA*24:02, conferred increased risk, some other alleles, such as A*24:07, did not. Since virtually all $\mathrm{A}^{* 24}$ alleles in Europeans are A*24:02, this limitation of the typing method should not affect the results. However, if this strategy were to be applied to other populations, a higher resolution typing system would be warranted. A significant strength of this study is that 5- and 10-year progression could be followed. The authors conclude that 


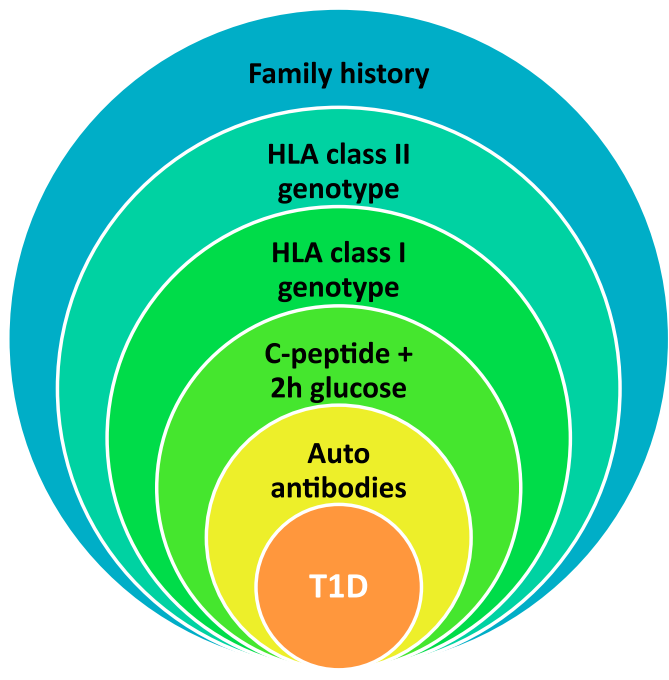

FIG. 1. Incorporating an increasing number of genetic, autoantibody, and metabolic markers in a predictive algorithm can identify a subset of the population at very high risk of T1D but at the cost of identifying only a small proportion of future T1D cases.

$\mathrm{A} * 24$ is an independent predictor of progression to T1D in antibody-positive FDR and that "depending on whether the screening goals intend to favor specificity or sensitivity, HLA-A $* 24$ can help select a subgroup at extremely high risk or increase sensitivity of detecting relatives with high combined Ab- and HLA-inferred risk." Clearly, other markers could be implemented into these predictive algorithms but the trade-off between sensitivity and specificity will, as in this study, have to be weighed.

The predictive power of a given diagnostic is usually summarized by a receiver operating characteristic curve, in which subjects are ranked in descending order of their predicted risk, and the cumulative proportion of subjects who develop disease (cases) is plotted against the corresponding cumulative proportion of the population, i.e., the sensitivity (true-positive fraction) is plotted in the $y$-axis versus 1-specificity (the false-negative fraction) in the $x$ axis (20). A formal receiver operating characteristic analysis of class II and class I HLA alleles, with or without dynamic biomarkers, has yet to be performed; however, the incorporation of additional markers, such as HLA-A*24 described by Mbunwe et al., into a predictive algorithm that can increase the positive predictive value for progression to T1D, promises to facilitate the long-standing goal of T1D prevention. Therapeutic interventions are likely to be more effective when introduced during the preclinical diabetes phase, characterized by autoimmunity while residual $\beta$-cell function is present. For these preventive strategies to succeed, identification of subjects at very high risk of T1D is critical. This publication represents another step toward achieving this important goal.

\section{ACKNOWLEDGMENTS}

H.A.E. is employed by Roche Molecular Systems, Pleasanton, California. No other potential conflicts of interest relevant to this article were reported.

\section{REFERENCES}

1. Concannon P, Erlich HA, Julier C, et al.; Type 1 Diabetes Genetics Consortium. Type 1 diabetes: evidence for susceptibility loci from four genomewide linkage scans in 1,435 multiplex families. Diabetes 2005;54:2995-3001

2. Erlich H, Valdes AM, Noble J, et al.; Type 1 Diabetes Genetics Consortium. HLA DR-DQ haplotypes and genotypes and type 1 diabetes risk: analysis of the Type 1 Diabetes Genetics Consortium families. Diabetes 2008;57:10841092

3. Noble JA, Valdes AM, Varney MD, et al.; Type 1 Diabetes Genetics Consortium. HLA class I and genetic susceptibility to type 1 diabetes: results from the Type 1 Diabetes Genetics Consortium. Diabetes 2010;59:2972-2979

4. Varney MD, Valdes AM, Carlson JA, et al.; Type 1 Diabetes Genetics Consortium. HLA DPA1, DPB1 alleles and haplotypes contribute to the risk associated with type 1 diabetes: analysis of the Type 1 Diabetes Genetics Consortium families. Diabetes 2010;59:2055-2062

5. Howson JM, Walker NM, Clayton D, Todd JA, Type 1 Diabetes Genetics Consortium. Confirmation of HLA class II independent type 1 diabetes associations in the major histocompatibility complex including HLA-B and HLA-A. Diabetes Obes Metab 2009;11(Suppl. 1):31-45

6. Nejentsev S, Howson JM, Walker NM, et al.; Wellcome Trust Case Control Consortium. Localization of type 1 diabetes susceptibility to the MHC class I genes HLA-B and HLA-A. Nature 2007;450:887-892

7. Knip M, Siljander H. Autoimmune mechanisms in type 1 diabetes. Autoimmun Rev 2008;7:550-557

8. LaGasse JM, Brantley MS, Leech NJ, et al. Successful prospective prediction of type 1 diabetes in schoolchildren through multiple defined autoantibodies: an 8-year follow-up of the Washington State Diabetes Prediction Study. Diabetes Care 2002;25:505-511

9. Parikka V, Näntö-Salonen K, Saarinen M, et al. Early seroconversion and rapidly increasing autoantibody concentrations predict prepubertal manifestation of type 1 diabetes in children at genetic risk. Diabetologia 2012; 55:1926-1936

10. Pietropaolo M, Towns R, Eisenbarth GS. Humoral autoimmunity in type 1 diabetes: prediction, significance, and detection of distinct disease subtypes. Cold Spring Harb Perspect Med. 2012;2:a012831

11. Barrett JC, Clayton DG, Concannon P, et al.; Type 1 Diabetes Genetics Consortium. Genome-wide association study and meta-analysis find that over 40 loci affect risk of type 1 diabetes. Nat Genet 2009;41:703-707

12. Aly TA, Ide A, Jahromi MM, et al. Extreme genetic risk for type 1A diabetes. Proc Natl Acad Sci USA 2006;103:14074-14079

13. Steck AK, Wong R, Wagner B, et al. Effects of non-HLA gene polymorphisms on development of islet autoimmunity and type 1 diabetes in a population with high-risk HLA-DR,DQ genotypes. Diabetes 2012;61:753758

14. Xu P, Beam CA, Cuthbertson D, Sosenko JM, Skyler JS, Krischer JP, DPT-1 Study Group. Prognostic accuracy of immunologic and metabolic markers for type 1 diabetes in a high-risk population: receiver operating characteristic analysis. Diabetes Care 2012;35:1975-1980

15. Yu L, Boulware DC, Beam CA, et al.; Type 1 Diabetes TrialNet Study Group. Zinc transporter- 8 autoantibodies improve prediction of type 1 diabetes in relatives positive for the standard biochemical autoantibodies. Diabetes Care 2012;35:1213-1218

16. Barker JM, Barriga KJ, Yu L, et al.; Diabetes Autoimmunity Study in the Young. Prediction of autoantibody positivity and progression to type 1 diabetes: Diabetes Autoimmunity Study in the Young (DAISY). J Clin Endocrinol Metab 2004;89:3896-3902

17. Mbunwe E, Van der Auwera BJ, Vermeulen I, et al. $H L A-A * 24$ is an independent predictor of 5-year progression to diabetes in autoantibodypositive first-degree relatives of type 1 diabetic patients. Diabetes 2013;62: $1345-1350$

18. Noble JA, Valdes AM, Bugawan TL, Apple RJ, Thomson G, Erlich HA. The HLA class I A locus affects susceptibility to type 1 diabetes. Hum Immunol 2002;63:657-664

19. Bugawan TL, Klitz W, Alejandrino M, et al. The association of specific HLA class I and II alleles with type 1 diabetes among Filipinos. Tissue Antigens 2002;59:452-469

20. Bossuyt P. Evidence-Based Laboratory Medicine: Principles, Practice, and Outcomes. Washington, DC, AACC Press, 2007, p. 67-81 